\title{
MANTENIMIENTO AUTOMOTRIZ BASADO EN UN DIAGNOSTICO TRIBOLÓGICO
}

\section{AUTOMOTIVE MAINTENANCE BASED ON A TRIBOLOGICAL DIAGNOSIS}

\author{
Ing. Miguel Ángel Olivares Torres*, PhD. Andrés López Velázquez \\ PhD. Rosario Aldana Franco*, PhD. Ervin Jesús Alvarez Sánchez*, Dr. \\ Fernando Aldana Franco ${ }^{\star *}$, Dra. Yazmín Rivera Peña*. Dr. José Gustavo \\ Leyva Retureta*
}

Universidad Veracruzana, Facultad de Ingeniería Mecánica y Eléctrica, Camp Xalapa, Cuerpo Académico Ingeniería Transdisciplinar.

Zircuito Dr. Gonzalo Aguirre Beltrán S/N, C.P. 91000, Zona Universitaria, Xalap

Veracruz, México.

Teléfono (+52) (228) 8421757 Ext. 11642.

E-mail: legna tovares@hotmail.com , andreslv66@hotmail.com , andlopez@uv.mx , raldana@uv.mx, eralvarez@uv.mx, faldana@uv.mx, yrivera@uv.mx, guleyva@uv.mx

Resumen: En este trabajo se presenta un plan de mantenimiento automotriz basado en un diagnóstico tribológico del aceite lubricante de origen mineral grado SAE 20W50 multigrado, utilizado en la lubricación de un motor de combustión interna de cuatro cilindros, $1800 \mathrm{~cm}^{3}$, motor en posición diagonal, enfriado por anticongelante, con un sistema de lubricación por bomba mecánica. Las muestras se obtuvieron bajo diferentes condiciones de uso: $0 \mathrm{~km}, 1000 \mathrm{~km}, 5000 \mathrm{~km}$ y $8500 \mathrm{~km}$; mismas que se sometieron a diversos ensayos, tales como: Viscosidad cinemática a $40^{\circ} \mathrm{C}$ y $100^{\circ} \mathrm{C}$ (Norma ASTMD445), Desgaste (Norma ASTM G99-05), Timken (Norma ASTM-D-2782-17) y Análisis de Aceite; esto con la finalidad de diagnosticar la degradación del aceite lubricante y el desgaste generado, considerando estos resultados, se formuló un plan de mantenimiento automotriz basado en un diagnostico tribológico del aceite lubricante del motor en estudio.

Palabras clave: Mantenimiento Automotriz, Lubricantes, Desgaste, Diagnostico Tribológico.

Abstract: This paper presents an automotive maintenance plan based on a tribological diagnosis of lubricating oil of mineral origin SAE $20 W 50$ multigrade grade, used in the operation of a four-cylinder internal combustion engine, $1800 \mathrm{~cm} 3$, engine diagonally, cooled by antifreeze, with a lubrication system by mechanical pump. The samples were obtained under different conditions of use: $0 \mathrm{~km}, 1000 \mathrm{~km}, 5000 \mathrm{~km}$ and $8500 \mathrm{~km}$; which were subjected to various tests, such as: Kinematic viscosity at $40^{\circ} \mathrm{C}$ and $100^{\circ} \mathrm{C}$ (Standard ASTMD445), Wear (Standard ASTM G99-05), Timken (Standard ASTM-D-2782-17) and Analysis of oil; this in order to diagnose the degradation of the lubricating oil and the wear generated, considering these results, an automotive maintenance plan was formulated based on a tribological diagnosis of the lubricating oil of the engine under study.

Keywords: Automotive Maintenance, Lubricants, Wear, Tribological Diagnosis.

\section{INTRODUCCIÓN}


Los motores de combustión interna son un tipo de máquinas térmicas que su funcionamiento se basa en obtener energía mecánica a partir de la energía interna liberada por el combustible al quemarse en su interior (Giacosa, 2000; Higuera et al, 2018). Este tipo de motores también es referido como alternativos y se clasifican de acuerdo con su ciclo termodinámico en: Motores de dos y cuatro tiempos (Alvarez, 2010). Cualquiera de estos motores puede ser operado a gasolina o Diesel. (Área tecnologica, 2010; Kristinne et al, 2015; Montalvo et al, 2018).

Las aplicaciones más relevantes de los motores a gasolina de dos tiempos han sido en motores pequeños como motosierras y podadoras (Widman International SRL, 2018). En el caso de los motores a Diesel de dos tiempos sus aplicaciones radican en los motores navales de gran potencia (Giacosa, 2000). En relación con las aplicaciones principales de los motores de cuatro tiempos tanto a gasolina como a Diesel es en el transporte terrestre (Área tecnologica, 2010; Salamanca et al, 2018).

El mantenimiento que requieren este tipo de motores para su correcto funcionamiento depende en gran medida de una adecuada lubricación (Lublearn, Noria, 2016; 2018; Motor, 2015 y Lima 2016; Rua et al, 2018). Por lo que en este trabajo se propone una metodología de mantenimiento automotriz basado en un diagnóstico tribológico que optimice la vida útil del aceite lubricante en el motor.

\section{METODOLOGIA}

La metodología para el desarrollo de este diagnóstico se basó en lo siguiente: Medición de viscosidad, análisis del aceite lubricante, desempeño lubricante y resistencia de la película lubricante (ASTM-D-2782-17) (Universidad
Veracruzana, 2016), y ensayos de desgaste (ASTM G99) (Universidad Veracruzana, 2016) de un aceite lubricante automotriz, en distintos momentos de uso (kilometraje), con el propósito de establecer un plan de mantenimiento automotriz basado en un diagnostico tribológico.

\section{a) Medición de viscosidad}

La medición de viscosidad del aceite lubricante se determinó a $40^{\circ} \mathrm{C}$ y $100^{\circ} \mathrm{C}$; con el propósito de verificar el estado de dicho parámetro, para poder correlacionarlo con el desempeño lubricante del mismo (Lublearn, Noria, 2016; Mora et al, 2018).

\section{b) Análisis del aceite lubricante.}

Para la determinación de las condiciones del aceite en este rubro, se utilizó el analizador de aceites Oilview Quick-check. Los aspectos del análisis del lubricante se enfocaron en tres áreas críticas de los aceites lubricantes:

$\checkmark$ Degradación química.

$\checkmark$ Contaminación por agua.

$\checkmark$ Presencia de partículas por desgaste.

\section{c) Ensayo Timken, ASTM-D-2782-17}

Esta característica se determinó de acuerdo con la norma ASTM-D-2782-17, ensayo Timken. El equipo que se utilizó fue una maquina Timken de la marca KILL A WATT como se muestra en la figura 1 , para lo cual se emplearon esferas de acero inoxidable AISI 420 grado 1000 que se limpian con keroseno para la eliminación de impurezas.

Para la realización de estos ensayos se utilizó una muestra $10 \mathrm{ml}$ de aceite lubricante. Este ensayo consiste en colocar una esfera de acero inoxidable en el extremo del brazo, a través del cual se aplicará una fuerza. En el otro extremo del brazo se colocan cargas para aplicar $10 \mathrm{~kg}$ durante 10 minutos a una velocidad de 
800 rpm, se toma la medición de la temperatura inicial y la temperatura final alcanzada en el ensayo.

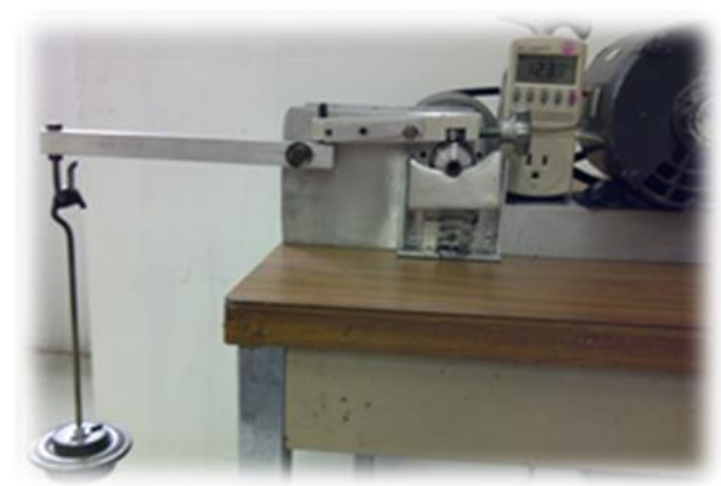

Figura 1. Máquina Timken. Laboratorio de Tribología, FIME-Xalapa. Universidad Veracruzana (Fuente propia).

Para el rompimiento de la película, se coloca una carga de $25 \mathrm{~kg}$ en el brazo de carga y cada minuto se va incrementando $5 \mathrm{~kg}$, se registra la temperatura en el contacto cada minuto, el rompimiento de la película se detecta de manera audible y cuando la maquina se amarra, se debe de apagar el equipo, parar el tiempo y realizar la medición de la temperatura que se alcanzó al final del ensayo (ASTM-D-278217) (Universidad Veracruzana, 2016). La máquina que se utilizó para estos ensayos es un tribómetro tipo pin sobre disco, del Laboratorio de Tribología de la FIMEXalapa (Figura 2).

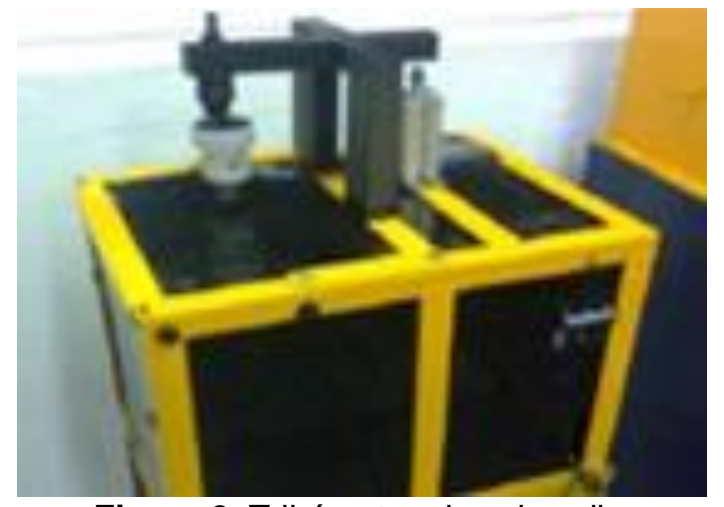

Figura 2. Tribómetro pin sobre disco.

Laboratorio de Tribología, FIME-Xalapa.

Universidad Veracruzana (Fuente propia).

d) Ensayo de desgaste
El método empleado para la determinación del desgaste fue del tipo pin sobre disco bajo la Norma ASTM G99-05, (Universidad Veracruzana, 2016). En términos generales el ensayo consiste en un pin el cual se coloca perpendicularmente a la otra probeta, generalmente un disco circular plano. El pin se presiona contra el disco a una carga específica. La máquina de ensayo permite que el disco gire y el pin presione el disco, como consecuencia se obtiene un camino o marca de desgaste sobre el disco.

Los ensayos se realizaron de acuerdo con las condiciones indicadas en la tabla 1.

Tabla 1. Matriz Experimental.

\begin{tabular}{ccc}
\hline $\begin{array}{l}\text { CORRIDA } \\
\text { EXP. }\end{array}$ & CARGA[N] & VELOCIDAD[RPM] \\
\hline 1 & 30 & 300 \\
2 & 30 & 1000 \\
3 & 100 & 300 \\
4 & 100 & 1000 \\
\hline (Fuente propia) &
\end{tabular}

\section{RESULTADOS}

En la figura 3 se muestran los resultados obtenidos de la medición de viscosidad, donde se resalta la gran diferencia entre los valores de viscosidad obtenidos a las temperaturas de $40^{\circ} \mathrm{C}$ y $100^{\circ} \mathrm{C}$. El incremento de la viscosidad a $40^{\circ} \mathrm{C}$ es más notorio, conforme se incrementa el kilometraje de trabajo. Sin embargo, para el caso de la viscosidad a $100^{\circ} \mathrm{C}$, prácticamente, la viscosidad permanece constante independientemente del kilometraje y esto es lo deseado para garantizar una película lubricante entre los componentes a lubricar.

La temperatura de trabajo recomendada para el aceite lubricante en motores de combustión interna es en el rango de $90^{\circ} \mathrm{C}$ a $100^{\circ} \mathrm{C}$ y mientras más degradado se encuentre el aceite lubricante, retiene mayor calor y ese exceso de calor afecta al motor. 


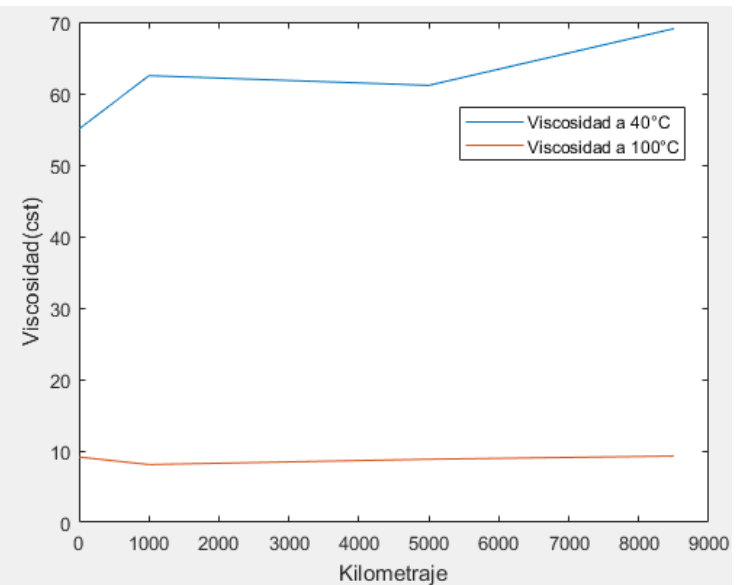

Figura 3. Comportamientos de la viscosidad cinemática del aceite $20 \mathrm{~W} 50$ a las temperaturas de $40^{\circ} \mathrm{C}$ y $100^{\circ} \mathrm{C}$ Vs. Kilometraje (Fuente propia).

Conforme va trabajando el aceite lubricante dentro de la máquina, el aceite se va oxidando, cada vez más, y a su vez, se van generando más contaminantes, los cuales hacen que incremente su viscosidad (Velandia et al, 2016).

En la figura 4 se presentan los resultados obtenidos a través del analizador de aceites Oilview Quick-check. Donde se aprecia que el índice dieléctrico del aceite lubricante $S A E \quad 20 W 50$ se va incrementando conforme éste trabaja en el motor. Esto debido a que el aceite se va oxidando y degradando químicamente. A pesar de ello, en este ensayo no se encontró evidencias de partículas de desgaste, sólo se evidencia un desgaste químico del aceite lubricante por su uso.

Todas las muestras de los aceites lubricantes ensayados cumplen con su desempeño lubricante adecuado, sin embargo, la resistencia al rompimiento de la película de estos varía dependiendo de las condiciones de carga y tiempo de su resistencia.

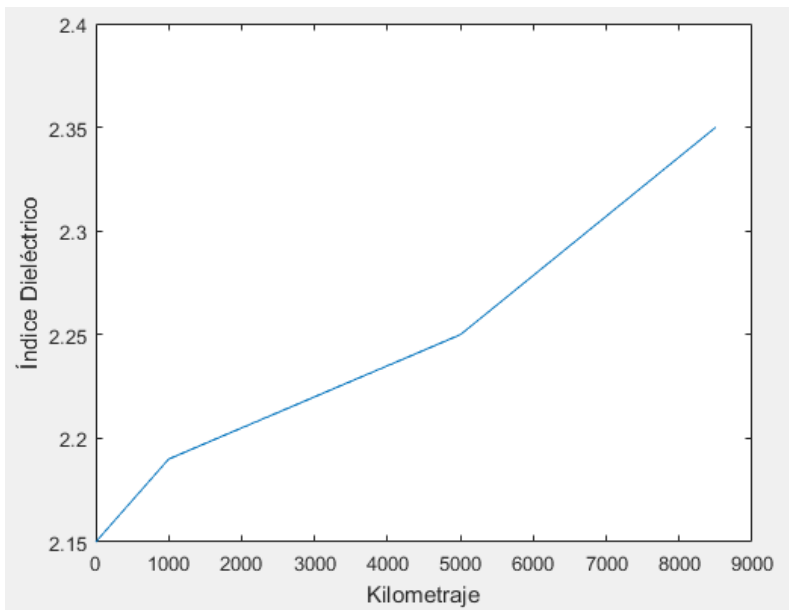

Figura 4. Comportamiento del índice dieléctrico del aceite $20 \mathrm{~W} 50$ Vs. Kilometraje (Fuente propia).

En la Tabla 2, se presentan los resultados obtenidos respecto del ensayo de rompimiento de la película lubricante del aceite SAE $20 W 50$ en sus diferentes condiciones de uso.

Tabla 2. Condiciones del rompimiento de la película lubricante del aceite $20 \mathrm{~W} 50 \mathrm{Vs}$. Kilometraje.

\begin{tabular}{lcccc}
$\begin{array}{l}\text { KILOMETRAJE } \\
{[\mathrm{Km}]}\end{array}$ & 0 & 1000 & 5000 & 8500 \\
\hline
\end{tabular}

\section{Carga al} rompimiento [Kg]

Tiempo del rompimiento [MINUTOS]

(Fuente propia)

Se determinó que las condiciones del rompimiento de la película de las muestras correspondientes a un kilometraje de $0 \mathrm{Km}$ (aceite nuevo) fueron a una carga de 65 $\mathrm{Kg}$ a un tiempo de 8:15 minutos y para las muestras a $8,500 \mathrm{Km}$ el rompimiento de la película se presentó a una carga de $60 \mathrm{Kg}$ en un tiempo de 8:00 minutos, lo cual refleja un ligero adelgazamiento de la película lubricante en las muestras de mayor kilometraje. 
De los resultados obtenidos en los ensayos de desgaste, (figura 5), se observa que el aceite a $0 \mathrm{Km}$ fue el que presento el menor desgaste, esto debido a que no contiene presencia de contaminantes y se encuentra en estado de nuevo. En el rango de $1,000 \mathrm{~km}$ a $5,000 \mathrm{~km}$, se observa que conforme el aceite se pone a trabajar llega a un momento en que optimiza su función lubricante, debido a que sus aditivos se activan de manera plena, lo cual corresponde a la vida útil del aceite lubricante. Después de $5,000 \mathrm{~km}$, el aceite lubricante se va degradando, con su uso, hasta que sus aditivos se agotan y sus propiedades lubricantes se pierden, presentándose un desgaste acelerado $(8,500 \mathrm{~km})$.

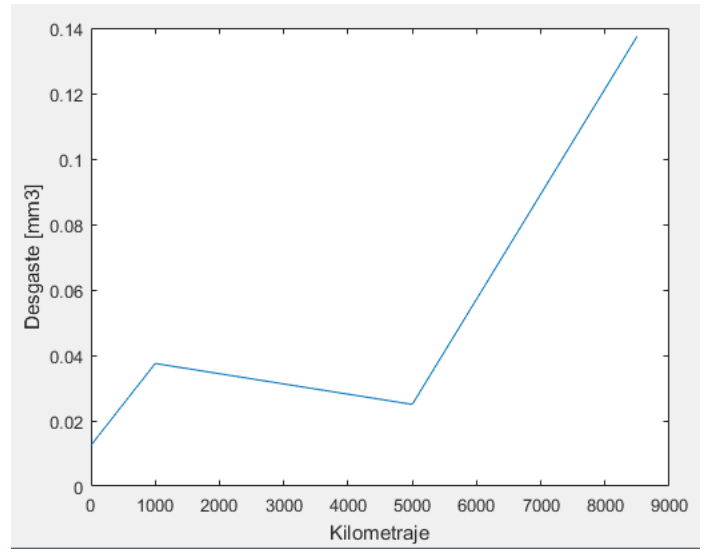

Figura 5. Comportamiento del desgaste lubricado con aceite lubricante $20 \mathrm{~W} 50$ Vs.

Kilometraje. Ensayo pin sobre disco (Fuente propia).

Con base en la información que nos ofrecen los ensayos tribológicos desarrollados en este trabajo, se propone el siguiente plan de mantenimiento automotriz:

1. Lo primero que se recomienda es realizar el cambio total del aceite lubricante del motor. Con el propósito de contar con una referencia de seguimiento para el mantenimiento, (0 $\mathrm{Km})$.
2. Transcurrido el primer bimestre, se procede a realizar los ensayos tribológicos, como se indican en la figura 6. Viscosidad a $100^{\circ} \mathrm{C}$, Índice dieléctrico, resistencia de película y desgaste.

3. En el segundo bimestre o posteriores, se propone realizar sólo el ensayo de viscosidad; si el resultado se encuentra dentro de los límites permisibles (8.40 cst $-10.51 \mathrm{cst}$ ), se recomienda continuar utilizando el aceite lubricante, sin necesidad de realizar algún otro ensayo tribológico, y al siguiente bimestre comenzar con el paso 3; si no se cumple con los límites establecidos de viscosidad continuar con el paso 4.

4. Si el resultado del ensayo de viscosidad sale de los límites permisibles, se procede con la determinación del índice dieléctrico y continuar con el paso 5.

\begin{tabular}{|c|c|c|c|c|c|}
\hline \multicolumn{6}{|c|}{ Numero de unidad: } \\
\hline \multirow{2}{*}{ BIMESTRE } & Viscosidad & $\begin{array}{c}\text { Análisis } \\
\text { de aceite }\end{array}$ & Timken & Pin sobre disco & $\begin{array}{l}\text { Actividad } \\
\text { especifica }\end{array}$ \\
\hline & $100^{\circ} \mathrm{C}$ & Dieléctrico & $\begin{array}{l}\text { Desgaste } \\
\text { Destructiva }\end{array}$ & $1000 \mathrm{rpm}$ a $30 \mathrm{~N}$ & \\
\hline \multicolumn{6}{|l|}{$\begin{array}{c}\text { PRIMER } \\
\text { BIMESTRE }\end{array}$} \\
\hline \multicolumn{6}{|l|}{$\begin{array}{l}\text { SEGUNDO } \\
\text { BIMESTRE }\end{array}$} \\
\hline \multicolumn{6}{|l|}{$\begin{array}{c}\text { TERCER } \\
\text { BIMESTRE }\end{array}$} \\
\hline \multicolumn{6}{|l|}{$\begin{array}{l}\text { CUARTO } \\
\text { BIMESTRE }\end{array}$} \\
\hline \multicolumn{6}{|l|}{$\begin{array}{l}\text { QUINTO } \\
\text { BIMESTRE }\end{array}$} \\
\hline $\begin{array}{l}\text { SEXTO } \\
\text { BIMESTRE }\end{array}$ & & & & & \\
\hline
\end{tabular}

Figura 6. Bitácora bimestral de mantenimiento automotriz (Olivares, 2016).

5. Si el resultado del análisis de aceite lubricante indica un incremento en el índice dieléctrico mayor a 0.03 comparado con el resultado del primer bimestre, realizar el paso 6; si el incremento del índice dieléctrico es menor a 0.03 se recomienda continuar utilizando el aceite lubricante, y en el siguiente bimestre comenzar en el paso 3.

6. Realizar el análisis de aceite, si los resultados muestran contaminación por agua, la actividad específica que se 
propone es cambiar el aceite lubricante inmediatamente y se procede desde el paso 1; pero si muestra degradación química, entonces, realizar el paso 7. En caso contrario, se seguir utilizando el aceite lubricante, $y$ al siguiente, bimestre realizar el paso 3 .

7. Realizar el ensayo Timken, si el resultado de la carga del rompimiento de película es menor a $60 \mathrm{~kg}$ y el tiempo del rompimiento de película es menor a 8:00 minutos, realice el paso 8; si el rompimiento de película lubricante se alcanza a una carga mayor a $60 \mathrm{~kg}$ y a un tiempo mayor a 8:00 minutos, continuar utilizando el mismo aceite lubricante, y al siguiente bimestre comenzar en el paso 3 .

8. Realizar los ensayos de desgaste pin sobre disco del aceite lubricante en función del bimestre, para evaluar el volumen de desgaste generado, si el volumen es mayor o igual a 0.0625 $\mathrm{mm}^{3}$ se procede a remplazar el aceite lubricante; en caso contrario, se continúa utilizando el aceite lubricante.

\section{CONCLUSIONES}

Se realizaron ensayos tribológicos de diferentes muestras del aceite lubricante SAE 20W50 multigrado, utilizado en la lubricación de un motor de combustión interna de cuatro cilindros. Las muestras se obtuvieron bajo diferentes condiciones de uso: 0 km, 1000 km, 5000 km y 8500 $\mathrm{km}$;

Se estableció una metodología de diagnóstico tribológico con base en cuatro ensayos, los cuales son: viscosidad, análisis de aceite, rompimiento de película y desgaste. Estos ensayos son útiles para establecer parámetros tribológicos del aceite lubricante y así poder determinar el estado de su vida útil.

Con base en esta metodología de diagnóstico tribológico, se propone un plan de mantenimiento automotriz, a través del cual se determine el momento justo $y$ necesario para realizar el cambio del aceite lubricante del motor, optimizándose así, el desempeño del aceite lubricante y reduciéndose el volumen de aceite lubricante usado y los costos del mantenimiento.

\section{RECONOCIMIENTO}

Al personal de apoyo y adjunto del Laboratorio de Tribología de la Facultad de Ingeniería Mecánica y Eléctrica, Región Xalapa de la Universidad Veracruzana. Circuito Dr. Gonzalo Aguirre Beltrán, S/N, Zona Universitaria, Ciudad de Xalapa, Veracruz, México, por su disposición y apoyo para la realización de los ensayos tribológicos.

\section{REFERENCIAS}

Alvarez F, J. (2010). Motores alternativos de combustion interna (1 ed.). España: Upc. Área tecnologica. (1 de Febrero de 2010). Tecnología., de partes del motor: http://www.areatecnologia.com/TUTORIALES/MOTOR\%20DE\%20COMBUSTION.htm (Consultado el 29 de Agosto de 2018).

Giacosa, D. (2000). Motores endotérmicos. España: Omega. Lima, A. (14 de Mayo de 2016). Doctor auto. mantenimiento previo automotriz: http://www.doctorauto.com.mx/2016/05/14/mantenimiento-preventivo- automotriz/ (Consultado el 20 de Agosto de 2018). 
Gutierrez, T., Castellanos, C. y Hernández, N. (2016). El ordenamiento territorial frente a las consecuencias de los cambios climáticos. Revista Ambiental Agua, Aire y Suelo. ISSN $1900-$ 9178. Volumen (7), Numero (2). DOI: https://doi.org/10.24054/19009178.v2.n2.2016.3338

Higuera, F., Salamanca, J. R., Acosta, L. F. y Acero, M. (2018). Análisis de acero laminado antes y después de soldado, mediante pruebas de metalografía y macroataque. Revista Ambiental Agua, Aire y Suelo. Volumen (9), Numero (1). DOI: https://doi.org/10.24054/19009178.v1.n1.2018.3209

Ivanova, Y. y Sarmiento, A. (2014). Evaluación de la huella hídrica de la ciudad de Bogotá como una herramienta de la gestión del agua en el territorio urbano. Revista Ambiental Agua, Aire y Suelo. ISSN 1900-9178. Volumen (4), Numero (2), 1-5.

DOI: https://doi.org/10.24054/19009178.v2.n2.2013.427

Kristinne Echávez, K., Pastran, Y. y Polo, A. (2015). Estimación del CO2 emitido y capturado en la sede sabanas y el campus deportivo de la Universidad Popular del Cesar. Revista Ambiental Agua, Aire y Suelo. ISSN 1900-9178. Volumen (6), Numero (2). DOI: https://doi.org/10.24054/19009178.v2.n2.2015.3252

Lublearn, Noria. (29 de Abril de 2016). Cómo medir la viscosidad en aceites industriales. ttp://noria.mx/lublearn/como-medir-la-viscosidad-en-aceites-industriales-y-de-motor/ (Consultado el 19 de Agosto de 2018).

Lublearn, Noria. (19 de enero de 2018). Cómo determinar la calidad del aceite del motor. http://noria.mx/lublearn/como-determinar-la-calidad-del-aceite-del-motor/(Consultado el 1 de septiembre de 2018). Motor. (12 de noviembre de 2015). El tiempo motor:http://www.motor.com.co/actualidad/industria/respuestas-10-preguntas-comuneslubricantes-motor/24222 (Consultado el 1 de septiembre de 2018).

Montalvo, A., Aldana, R., López, A., Álvarez, E., Aldana, F. y Rivera, Y. (2018). Mantenimiento centrado en confiabilidad en motocompresores. Revista Ambiental Agua, Aire y Suelo. ISSN 1900-9178. Volumen (9), Numero (1). DOI: https://doi.org/10.24054/19009178.v1.n1.2018.3212

Mora, E., Martínez, E. y Velasco, J. (2018). Simulación y validación del prototipo de un colector térmico solar hecho con neumáticos reciclados. Revista Ambiental Agua, Aire y Suelo. Volumen (9), Numero (2). DOI: https://doi.org/10.24054/19009178.v2.n2.2018.3218

Moreno, C. y Rueda, L. (2016). La educación ambiental como herramienta para la recuperación de la cobertura vegetal, mediante prácticas agro-ecológicas en la comunidad minera asograstorres, asociación de gravilleros de Sabana de Torres. Revista Ambiental Agua, Aire y Suelo. ISSN 1900-9178. Volumen (7), Numero DOI: https://doi.org/10.24054/19009178.v1.n1.2016.3260

Olivares, M. A. (2016). Plan de mantenimiento automotriz basado en un diagnostico tribologico. Xalapa, Veracruz. México: Universidad Veracruzana.

Salamanca, J., Acosta, L., Higuera, F. y Rojas, B. (2018). Desarrollo de chasis de un vehículo de tracción humana, a base de guadua y polímero. Revista Ambiental Agua, Aire y Suelo. Volumen (9), Numero (2). DOI: https://doi.org/10.24054/19009178.v2.n2.2018.3215 
Rua, E., Gonzales, A., Granados, A. y Ramírez, R. (2018). Diseño estructural de transporte para sistema de bombeo portátil activado con energía solar fotovoltaica para el departamento de Boyacá. Revista Ambiental Agua, Aire y Suelo. Volumen (9), Numero (2). DOI: https://doi.org/10.24054/19009178.v2.n2.2018.3219

Universidad Veracruzana. (2016). Manual de prácticas del labortorio de tribología. Xalapa, Veracruz. México: Universidad Veracruzana.

Velandia, F. J., Granados, J. D., Ramón, J. D. y Roa, A. L. (2016). Caracterización de consorcios microbianos con potencial degradador de contaminantes en el municipio de Pamplona, Norte de Santander. Revista Ambiental Agua, Aire y Suelo. ISSN 1900-9178. Volumen (7), Numero (1). DOI: https://doi.org/10.24054/19009178.v1.n1.2016.3278

Widman International SRL. (24 de Agosto de 2018). Mantenimiento proactico. Mantenimiento simple del auto para maximizar la Vida útil y minimizar costos: https://www.widman.biz/mantenimiento/autos.html (Consultado el 1 de septiembre de 2018). 